\title{
Replacing Leads by Self-Energies Using Nonequilibrium Green's Functions
}

\author{
Fredrick Michael and M.D. Johnson \\ Department of Physics, University of Central Florida, Orlando, FL 32816-2385
}

(Dated: March 22, 2002)

\begin{abstract}
An open quantum system consists of leads connected to a device of interest. Within the nonequilibrium Green's function technique, we examine the replacement of leads by self-energies in continuum calculations. Our starting point is a formulation of the problem for continuum systems by T.E. Feuchtwang. In this approach there is considerable flexibility in the choice of unperturbed Green's functions. We examine the consequences of this freedom on the treatment of leads. For any choice the leads can be replaced by coupling self-energies which are simple functions of energy. We find that the retarded self-energy $\Sigma^{r}$ depends on the details of the choice of unperturbed Green's function, and can take any value. However, the nonequilibrum self-energy or scattering function $\Sigma^{<}$can be taken to be independent of this choice. Expressed in terms of these self-energies, nonequilibrium transport calculations take a particularly simple form.
\end{abstract}

PACS numbers: 73.23.-b, 73.63.-b,73.40.-c 


\section{INTRODUCTION}

A general method for calculating electronic transport properties is provided by the use of non-equilibrium Green's functions (NEGF). 1 . and non-equilibrium formulation, and because its foundational assumptions are transparent, this technique has been used to study a very wide range of transport problems.

In this paper we will examine the way in which the leads permitting transport through an open quantum system (a "device") can be replaced by self-energy terms in a Dyson's equation. The analysis is limited to the case where the leads can be well-approximated as systems of non-interacting electrons in local thermodynamic equilibrium. In principle this approximation is not very restrictive, since sufficiently far even from a mesoscopic device the wires carrying current become macroscopic. Notice that the device whose transport properties are being calculated is left completely unspecified - it can contain interactions, disorder, etc.

A similar treatment of the leads has been used by Datta, in an approximation in which the spatial continuum is replaced by a lattice of discrete points.6 An apparently very different approach for continuum systems was developed in a series of papers by Caroli and co-workers calculations by, for example, Ref. 9. Other workers have also cast the continuum problem into a self-energy form.11

Our point here is to show that the Feuchtwang approach for the continuum also can be repackaged so that leads are replaced exactly by simple self-energies, and to explore this possibility in some detail. We make two simple changes to Feuchtwang's calculation: regrouping the Dyson's equations to eliminate differential operators, and then using analytic continuation as provided by Langreth's theorem目 (and learned by us from Haug and Jaughol we will refer to this admirable textbook for all standard information about NEGF). The result is in our opinion pleasingly clear, and simplifies transport calculations of electronic devices.

There is some freedom in how the self-energy is identified. A Hamiltonian $H$ can in principle be arbitrarily separated into an unperturbed $H_{0}$ and a perturbation $H-H_{0}$. If calculations can then be carried out exactly, the results will be independent of the initial separation. There is a similar arbitrariness in the definition of the unperturbed retarded 
$g^{r}$ and its corresponding retarded self-energy $\Sigma^{r}$. (In the Feuchtwang approach this comes from an arbitrary choice of internal boundary conditions). The exact Green's functions are of course independent of this choice. It turns out, moreover, that the "less-than" self-energy $\Sigma^{<}$is independent of this choice, for a broad class of internal boundary conditions.

For clarity we will focus on the simplest case: a one-dimensional system of non-interacting, spinless electrons in steady-state. But the results are rather general. For example, electronelectron and other interactions can be included by adding their appropriate self-energies to the coupling self-energy described here.

Extension to two and three dimensions is also straightforward. One motivation for this paper is that the approach we describe turns out to work well even for systems with irregular shapes 10 Because the final results are independent of the choice of internal boundary conditions, in practice one should make whatever choice is simplest. In one dimension any choice is equally easy, but in higher dimensions with irregular shapes, the right choice can simplify the calculation considerably.10

The model we will consider is an open one-dimensional system consisting of two leads $\mathrm{L}$ and $\mathrm{R}$ (at $x<L$ and $x>R$, respectively), connected to a device $\mathrm{D}$ (within $L<x<R$ ). This has Hamiltonian

$$
H=\theta(L-x) H_{L}+\theta(x-L) \theta(R-x) H_{D}+\theta(x-R) H_{R} .
$$

where $\theta$ is a step function ( 0 to 1$)$ and

$$
H_{\alpha}=-\frac{\hbar^{2}}{2 m} \frac{\partial^{2}}{\partial x^{2}}+V_{\alpha}(x), \quad \alpha=L, D, R .
$$

The single-particle potential energies $V_{\alpha}(x)$ are arbitrary. To give definite examples in the following we will usually assume that $V_{L, R}$ (but not $V_{D}$ ) are constants. The leads are treated as reservoirs of non-interacting electrons in local thermodynamic equilibrium described by local chemical potentials $\mu_{L}, \mu_{R}$. An applied bias $e V$ would be included by setting $\mu_{L}=\mu_{R}+e V$ and perhaps $V_{L}=V_{R}+e V$. This would lead to a current flow $I$, and a typical purpose of a NEGF calculation is to find the I-V characteristics of the device. The current and other physical observables of a non-equilibrium system are obtained from the total "less-than" function $G^{<}$. We concentrate here on finding $G^{<}$. The path we follow is to calculate the retarded Green's function $G^{r}$ and then use analytic continuation rules to obtain $G^{<}$. 


\section{RETARDED GREEN'S FUNCTION $G^{r}$}

When the Hamiltonian is independent of time, the retarded Green's function $G^{r}=$ $G^{r}\left(x_{1} t_{1}, x_{2} t_{2}\right)$ can be Fourier transformed in $t_{1}-t_{2}$. For brevity denote the result $G(12)=$ $G\left(x_{1} x_{2} \omega\right)$. For a system of noninteracting electrons this satisfies the equation of motion

$$
[\hbar \omega-H(1)+i \eta] G^{r}(12)=\delta\left(x_{1}-x_{2}\right)
$$

Here $H(1)$ is the total Hamiltonian in Eq. (11) written in terms of coordinate $x_{1}$, and $\eta>0$ is infinitesimal. The advanced Green's functions, used below, are simply obtained by $G^{a}(12)=$ $\left[G^{r}(12)\right]^{*}$.

\section{A. Unperturbed $g^{r}$}

Following Feuchtwang, Green's functions associated with each region $\alpha=L, D, R$ :

$$
\left[\hbar \omega-H_{\alpha}(1)+i \eta\right] g_{\alpha}^{r}(12)=\delta\left(x_{1}-x_{2}\right),
$$

subject to internal boundary conditions at $x=L, R$. Like Feuchtwang we will consider the set of possible homogeneous boundary conditions

$$
\begin{aligned}
& \left.\frac{\partial g_{L}^{r}(12)}{\partial x_{1}}\right|_{x_{1}=L}=\gamma_{L} g_{L}^{r}(L 2), \quad \text { if } x_{2}<L \\
& \left.\frac{\partial g_{D}^{r}(12)}{\partial x_{1}}\right|_{x_{1}=L}=\gamma_{D L} g_{D}^{r}(L 2), \quad \text { if } L<x_{2}<R \\
& \left.\frac{\partial g_{D}^{r}(12)}{\partial x_{1}}\right|_{x_{1}=R}=\gamma_{D R} g_{D}^{r}(R 2), \quad \text { if } L<x_{2}<R \\
& \left.\frac{\partial g_{R}^{r}(12)}{\partial x_{1}}\right|_{x_{1}=R}=\gamma_{R} g_{R}^{r}(R 2), \quad \text { if } x_{2}>R,
\end{aligned}
$$

plus identical conditions with $x_{1}$ and $x_{2}$ interchanged. Unlike Feuchtwang, we do not assume that the $\gamma$ are all equal. (This is important in higher dimensions.10) Eqs. (5) include Dirichlet and von Neumann boundary conditions as the limiting cases $\gamma \rightarrow \infty, 0$, respectively. The total Green's functions $G$ cannot, and as we will see do not, depend on these internal boundary conditions.

One also needs boundary conditions at the system's physical boundaries $x_{i} \rightarrow \pm \infty$. These can be any homogeneous conditions. As long as we choose $G$ to have the same homogeneous conditions on the physical boundaries, the boundary conditions there play no role. 
Both the perturbed and unperturbed retarded functions have matching conditions of the form

$$
\left.\frac{\partial g^{r}(12)}{\partial x_{1}}\right|_{x_{1}=x_{2}-\epsilon} ^{x_{2}+\epsilon}=\frac{2 m}{\hbar^{2}},\left.\quad g^{r}(12)\right|_{x_{1}=x_{2}-\epsilon} ^{x_{2}+\epsilon}=0
$$

for infinitesimal $\epsilon>0$. The first of these comes from integrating Eq. (雨) across an infinitesimal interval about $x_{1}=x_{2}$.

When $V_{L}$ and $V_{R}$ are constant, it is simple to obtain the unperturbed $g_{\alpha}^{r}$ in the leads $(\alpha=L, R)$. Solve Eq. (四) separately in the regions $x_{1}<x_{2}$ and $x_{1}>x_{2}$, and then use the boundary conditions Eq. (5a,d) and the matching conditions Eq. (6) to fix the undetermined constants. The infinitesimal $\eta$ in Eq. (4) serves to pick out the outgoing (retarded) solution and can then be set to zero. The results are

$$
\begin{aligned}
& g_{L}^{r}(12)=\frac{2 m}{\hbar^{2} k_{L}} e^{-i\left[k_{L}\left(x_{<}-L\right)+\phi_{L}\right]} \sin \left[k_{L}\left(x_{>}-L\right)+\phi_{L}\right] \\
& g_{R}^{r}(12)=-\frac{2 m}{\hbar^{2} k_{R}} e^{i\left[k_{R}\left(x_{>}-R\right)+\phi_{R}\right]} \sin \left[k_{R}\left(x_{<}-R\right)+\phi_{R}\right]
\end{aligned}
$$

where $x_{>}\left(x_{<}\right)$is the greater (lesser) of $x_{1}$ and $x_{2}$, and where

$$
\begin{aligned}
\hbar \omega & =V_{\alpha}+\frac{\hbar^{2} k_{\alpha}^{2}}{2 m}, \\
\gamma_{\alpha} & =k_{\alpha} \cot \phi_{\alpha},
\end{aligned}
$$

for $\alpha=L, R$. If $\gamma_{L, R}$ are real, so too are $\phi_{R, L}$. It is apparent in Eqs. (7) that the unperturbed $g^{r}(12)$ are symmetric under $x_{1} \leftrightarrow x_{2}$. This is true for all other Green's functions as well.

\section{B. Total $G^{r}$}

Once more following Feuchtwang, $\mathrm{B}$ consider the following integral over lead L:

$$
\begin{array}{r}
\int_{-\infty}^{L} d x_{3}\left\{G^{r}(32)\left[\hbar \omega-H_{L}(3)+i \eta\right] g_{L}^{r}(13)-g_{L}^{r}(13)[\hbar \omega-H(3)+i \eta] G^{r}(32)\right\} \\
=\theta\left(L-x_{1}\right) G^{r}(12)-\theta\left(L-x_{2}\right) g_{L}^{r}(12) .
\end{array}
$$

The equality follows from the equations of motion, Eqs. (3, (1). On the left-hand side of Eq. (9), all the terms in the two square brackets cancel except those involving derivatives. Hence the above integral also equals

$$
\begin{array}{r}
\frac{\hbar^{2}}{2 m} \int_{-\infty}^{L} d x_{3}\left(G^{r} \frac{\partial^{2} g_{L}^{r}}{\partial x_{3}^{2}}-g_{L}^{r} \frac{\partial^{2} G^{r}}{\partial x_{3}^{2}}\right) \\
=\frac{\hbar^{2}}{2 m}\left[G^{r}(32) \frac{\partial g_{L}^{r}(13)}{\partial x_{3}}-g_{L}^{r}(13) \frac{\partial G^{r}(32)}{\partial x_{3}}\right]_{x_{3}=L} .
\end{array}
$$


Here the right-hand side follows from Green's theorem. (There are no contributions from the physical boundary $x_{3} \rightarrow-\infty$ as long as $g_{L}^{r}$ and $G$ have identical homogeneous conditions there.) Equating the right-hand sides of Eqs. (9, 10), yields

$$
G^{r}(12)=g_{L}^{r}(12) \theta\left(L-x_{2}\right)+\frac{\hbar^{2}}{2 m}\left[\frac{\partial g_{L}^{r}(13)}{\partial x_{3}} G^{r}(32)-g_{L}^{r}(13) \frac{\partial G^{r}(32)}{\partial x_{3}}\right]_{x_{3}=L}\left(x_{1}<L\right) .
$$

We can perform similar integrals over the other two regions, replacing $g_{L}^{r}$ by $g_{D}^{r}$ or $g_{R}^{r}$ as appropriate, with the result:

$$
\begin{aligned}
G^{r}(12) & =g_{D}^{r}(12) \theta\left(x_{2}-L\right) \theta\left(R-x_{2}\right) \\
& +\frac{\hbar^{2}}{2 m}\left[\frac{\partial g_{D}^{r}(13)}{\partial x_{3}} G^{r}(32)-g_{D}^{r}(13) \frac{\partial G^{r}(32)}{\partial x_{3}}\right]_{x_{3}=L}^{R}\left(L<x_{1}<R\right) \\
G^{r}(12) & =g_{R}^{r}(12) \theta\left(x_{2}-R\right)-\frac{\hbar^{2}}{2 m}\left[\frac{\partial g_{R}^{r}(13)}{\partial x_{3}} G^{r}(32)-g_{R}^{r}(13) \frac{\partial G^{r}(32)}{\partial x_{3}}\right]_{x_{3}=R}\left(x_{1}>R\right)(11 \mathrm{c})
\end{aligned}
$$

Each of Eqs. (11) is of the form of a Dyson equation $\left(G^{r}=g^{r}+g^{r} \Sigma^{r} G^{r}\right)$. They can be assembled into a single Dyson's equation for all space. 8 However, the self-energies include differential operators, an unattractive feature.

\section{Replace the leads by coupling self-energies}

Eliminating the derivatives gives a simpler expression. This is where we diverge from Feuchtwang's path. Let us focus on the only case of interest, $G^{r}(12)$ when both arguments lie within the device $\left(L<x_{1}, x_{2}<R\right)$. This is given by Eq. (11]). Replacing $\partial g_{D}^{r} / \partial x_{3}$ at the internal boundaries using Eq. (巨b,c),

$$
\begin{aligned}
G^{r}(12)=g_{D}^{r}(12) & +\frac{\hbar^{2}}{2 m} g_{D}^{r}(1 R)\left[\gamma_{D R} G^{r}(R 2)-\left.\frac{\partial G^{r}(32)}{\partial x_{3}}\right|_{x_{3}=R}\right] \\
& -\frac{\hbar^{2}}{2 m} g_{D}^{r}(1 L)\left[\gamma_{D L} G^{r}(L 2)-\left.\frac{\partial G^{r}(32)}{\partial x_{3}}\right|_{x_{3}=L}\right]
\end{aligned}
$$

when $L<x_{1}, x_{2}<R$. Here we need $\partial G^{r}(32) / \partial x_{3}$ at $x_{3}=L^{+}$. This is obtained from Eq. (11a) by using Eq. (5a), choosing $x_{2}>L$, and letting $x_{1} \rightarrow L^{-}$:

$$
\left.\frac{\partial G^{r}(12)}{\partial x_{1}}\right|_{L^{-}}=\left(\gamma_{L}-\frac{2 m}{\hbar^{2}} \frac{1}{g_{L}^{r}(L L)}\right) G^{r}(L 2), \quad x_{2}>L .
$$


The total $G^{r}$ and its derivative are continuous across the internal boundaries. Consequently Eq. (13) (and a similar expression for the derivative at $R$ ) give

$$
\begin{aligned}
& \left.\frac{\partial G^{r}(32)}{\partial x_{3}}\right|_{L}=\left(\gamma_{L}-\frac{2 m}{\hbar^{2}} \frac{1}{g_{L}^{r}(L L)}\right) G^{r}(L 2), \quad x_{2}>L \\
& \left.\frac{\partial G^{r}(32)}{\partial x_{3}}\right|_{R}=\left(\gamma_{R}+\frac{2 m}{\hbar^{2}} \frac{1}{g_{R}^{r}(R R)}\right) G^{r}(R 2), \quad x_{2}<R .
\end{aligned}
$$

Plugging these derivatives into Eq. (12) gives the following Dyson equation for $G^{r}$ in the device:

$$
\begin{array}{r}
G^{r}(12)=g_{D}^{r}(12)+g_{D}^{r}(1 L) \Sigma_{L}^{r}(\omega) G^{r}(L 2)+g_{D}^{r}(1 R) \Sigma_{R}^{r}(\omega) G^{r}(R 2) \\
\left(L<x_{1}, x_{2}<R\right),
\end{array}
$$

where

$$
\begin{aligned}
\Sigma_{L}^{r}(\omega) & =-\frac{\hbar^{2}}{2 m}\left(\gamma_{D L}-\gamma_{L}\right)-\frac{1}{g_{L}^{r}(L L)}, \\
\Sigma_{R}^{r}(\omega) & =\frac{\hbar^{2}}{2 m}\left(\gamma_{D R}-\gamma_{R}\right)-\frac{1}{g_{R}^{r}(R R)} .
\end{aligned}
$$

Thus for the purpose of finding $G^{r}$ in the device, the contribution of the leads has been replaced by simple coupling self-energies $\Sigma_{L, R}^{r}$. Notice that these are now $c$-numbers and not operators. This result is correct even for Dirichlet conditions as long as they are obtained as the limit $\gamma_{\alpha} \rightarrow \infty$. One can get a final closed expression for $G^{r}(12)$ by evaluating Eq. (15) at $x_{1}=L, R$, solving the resulting pair of equations for $G^{r}(L 2), G^{r}(R 2)$, and substituting the results back into Eq. (15).

The standard case will have a constant potential $V_{L, R}$ in the two leads, in which case we can substitute the particular expressions Eqs. (7) into Eqs. (16):

$$
\begin{aligned}
& \Sigma_{L}^{r}(\omega)=\frac{\hbar^{2}}{2 m}\left(-\gamma_{D L}-i k_{L}\right) \\
& \Sigma_{R}^{r}(\omega)=\frac{\hbar^{2}}{2 m}\left(\gamma_{D R}-i k_{R}\right) .
\end{aligned}
$$

We see that the choice of boundary condition in the leads drops out completely — there is no dependence on $\gamma_{L, R}$ left in Eqs. (17). However, the retarded self-energies $\Sigma^{r}$ do depend on the boundary conditions for the unperturbed $g_{D}^{r}$ in the device, via $\gamma_{D L}$ and $\gamma_{D R}$. We emphasize again the total $G^{r}$ solved from Eq. (15) will be independent of this choice, also.

These results agree with the discretized formulation by Datta目 when the latter is taken to a continuum limit. Datta discretizes the coordinates into lattice points $j a$ (with $j$ an 
integer). In the Hamiltonian the kinetic energy term becomes a second difference. The step from the last site in lead $\mathrm{L}$ (at $L-a$, say) and the first site in the device (at $L$ ) becomes a perturbation. (A similar perturbation connects $R$ in the device to $R+a$ in lead R.) Then an analysis like the above shows that the contribution of lead L to the discrete $\tilde{G}^{r}$ in the device can be written in terms of a self-energy

$$
\tilde{\Sigma}_{L}^{r}=-\frac{\hbar^{2}}{2 m a^{2}} e^{i \tilde{k}_{L} a}
$$

where

$$
\hbar \omega=\frac{\hbar^{2}}{m a^{2}}\left(1-\cos \tilde{k}_{L} a\right)
$$

For small lattice constant $a, \tilde{k}_{L} \approx k_{L}$ and Eq. (18) becomes

$$
a \tilde{\Sigma}_{L}^{r} \approx \frac{\hbar^{2}}{2 m}\left(-a^{-1}-i k_{L}\right)
$$

In the discrete approximation the unperturbed $\tilde{g}_{D}^{r}$ in the device vanishes at $L-a$. Thus for small $a$,

$$
\tilde{g}_{D}^{r}\left(L-a, x_{2}\right)=0 \approx \tilde{g}_{D}^{r}\left(L x_{2}\right)-\left.a \frac{\partial \tilde{g}_{D}^{r}(12)}{\partial x_{1}}\right|_{x_{1}=L} .
$$

That is, the discrete calculation for small lattice constant $a$ is equivalent to homogeneous boundary conditions with $\gamma_{D L}=a^{-1}$. Then the discrete self-energy Eq. (20) agrees in the continuum limit with our general result Eq. (17a).

Because the boundary constants $\gamma_{D L}, \gamma_{D R}$ are arbitrary, one can obviously choose $\Sigma_{L, R}^{r}$ with complete freedom. Probably the simplest case is to have von Neumann boundary conditions in the device $\left(\gamma_{D L}=0=\gamma_{D R}\right)$. This choice is the only simple one in higher

dimensions for devices of varying cross section.10 But every other choice is also possible. One can even choose $\Sigma_{L, R}^{r}$ to vanish (in which case $\gamma_{D L}=-i k_{L}$ and $\gamma_{D R}=i k_{R}$ are purely imaginary). The latter case amounts to building the contribution of the leads into the unperturbed $g_{D}^{r}$.

\section{NONEQUILIBRIUM GREEN'S FUNCTION $G^{<}$}

In symbolic notation, the Dyson's equation Eq. (15) for $G^{r}$ in the device is

$$
G^{r}=g_{D}^{r}+g_{D}^{r} \Sigma^{r} G^{r}
$$


Standard analytic continuation rules function $G$ in the device satisfies

$$
G=g_{D}+g_{D} \Sigma G
$$

where $g_{\alpha}$ is an unperturbed contour-ordered Green's function and $\Sigma$ is the contour-ordered analogue of $\Sigma^{r}$. Then a further application of the analytic continuation rules yields

$$
G^{<}=g_{D}^{<}+g_{D}^{r} \Sigma^{r} G^{<}+g_{D}^{r} \Sigma^{<} G^{a}+g_{D}^{<} \Sigma^{a} G^{a} .
$$

Here $G^{a}(12)=\left[G^{r}(12)\right]^{*}$ is the advanced total Green's function and $\Sigma^{a}=\left(\Sigma^{r}\right)^{*}$ the advanced self-energy.

But what is the "less-than" self-energy $\Sigma^{<}$? Our expressions Eqs. (16) for the retarded self-energies $\Sigma_{L, R}^{r}$ involve inverses, and can't be analytically continued using the standard

rules.目 One could make the following physical argument:日 the self-energies considered here represent the coupling to leads which are in local equilibrium; hence one should be able to use the relationship between retarded and less-than functions valid for equilibrium quantities. That is,

$$
\Sigma_{\alpha}^{<}(\omega)=i f_{\alpha}(\omega) \Gamma_{\alpha}(\omega)
$$

where

$$
\Gamma_{\alpha}(\omega)=i\left[\Sigma_{\alpha}^{r}(\omega)-\Sigma_{\alpha}^{a}(\omega)\right]
$$

for $\alpha=L, R$. Here $f_{\alpha}(\omega)=1 /\left(\exp \left[\beta\left(\hbar \omega-\mu_{\alpha}\right)\right]+1\right)$ is the Fermi-Dirac equilibrium distribution for region $\alpha$. As long as $\gamma_{D L}, \gamma_{D R}$ are real, substituting Eqs. (17) into Eqs. (25) gives

$$
\Sigma_{\alpha}^{<}(\omega)=i f_{\alpha}(\omega) \frac{\hbar^{2} k_{\alpha}}{m} \theta\left(\hbar \omega-V_{\alpha}\right), \quad \alpha=L, R .
$$

While this physical argument may not be entirely convincing, it has the merit of being correct (for real $\gamma_{D L}, \gamma_{D R}$ ), as we will now show.

\section{A. Unperturbed $g^{<}$}

Before coupling the three regions, each is in local equilibrium with local chemical potential $\mu_{\alpha}(\alpha=L, D, R)$. Consequently we can use the equilibrium relationship between $g^{<}$and $g^{r}$ :

$$
g_{\alpha}^{<}(12)=i f_{\alpha}(\omega) A_{\alpha}(12)
$$


where the spectral function in each region is:

$$
A_{\alpha}(12)=i\left[g_{\alpha}^{r}(12)-g_{\alpha}^{a}(12)\right]
$$

When all of the $\gamma$ in Eqs. (5) are real, each $g_{\alpha}^{<}$satisfies boundary conditions exactly like those in Eqs. (5). We will restrict ourselves to this case until the end of this section.

When $V_{L, R}$ are constant, the unperturbed $g^{<}$in the leads $(\alpha=L, R)$ are

$$
g_{\alpha}^{<}(12)=i f_{\alpha}(\omega) \theta\left(\hbar \omega-V_{\alpha}\right) \frac{4 m}{\hbar^{2} k_{\alpha}} \sin \left[k_{\alpha}\left(x_{1}-\alpha\right)+\phi_{\alpha}\right] \sin \left[k_{\alpha}\left(x_{2}-\alpha\right)+\phi_{\alpha}\right]
$$

using Eqs. (77,27). The unperturbed $g_{D}^{<}$in the finite device is a sum over Dirac $\delta$-functions. We will show later that it plays no role.

\section{B. Analytic continuation}

We calculate the total $G^{<}$using the analytic continuation rules summarized by ${ }^{\mathrm{E}}$

$$
\begin{aligned}
& (A B)^{r} \rightarrow A^{r} B^{r} \\
& (A B)^{<} \rightarrow A^{r} B^{<}+A^{<} B^{a} .
\end{aligned}
$$

Here $A$ (and $B$ ) are contour-ordered quantities, and $A^{r}, A^{<}$are the retarded and less-than components of $A$. Begin with Eqs. (11). Using Eq. (29a), each of these can be analytically continued back to contour-ordered expressions. (Simply remove the superscript $r$ ). Then analytically continuing again with Eq. (29]b) we have

$$
\begin{aligned}
G^{<}(12)=\frac{\hbar^{2}}{2 m}[ & \frac{\partial g_{L}^{r}(13)}{\partial x_{3}} G^{<}(32)-g_{L}^{r}(13) \frac{\partial G^{<}(32)}{\partial x_{3}} \\
+ & \left.\frac{\partial g_{L}^{<}(13)}{\partial x_{3}} G^{a}(32)-g_{L}^{<}(13) \frac{\partial G^{a}(32)}{\partial x_{3}}\right]_{x_{3}=L} \quad\left(x_{1}<L, x_{2}>L\right), \\
G^{<}(12)=g_{D}^{<}(12)+\frac{\hbar^{2}}{2 m}\left[\frac{\partial g_{D}^{r}(13)}{\partial x_{3}} G^{<}(32)-g_{D}^{r}(13) \frac{\partial G^{<}(32)}{\partial x_{3}}\right. & \\
+ & \left.\frac{\partial g_{D}^{<}(13)}{\partial x_{3}} G^{a}(32)-g_{D}^{<}(13) \frac{\partial G^{a}(32)}{\partial x_{3}}\right]_{x_{3}=L}^{R} \quad\left(L<x_{1}, x_{2}<R\right), \\
G^{<}(12)=-\frac{\hbar^{2}}{2 m} & {\left[\frac{\partial g_{R}^{r}(13)}{\partial x_{3}} G^{<}(32)-g_{R}^{r}(13) \frac{\partial G^{<}(32)}{\partial x_{3}}\right.}
\end{aligned} \quad\left(x_{1}>R, x_{2}<R\right) .
$$


The derivatives here can be eliminated much as they were in the Dyson equation for $G^{r}$. Eqs. (30a,c) yield the derivatives $\partial G^{<} / \partial x$ at $L, R$. The complex conjugate of Eqs. (14) gives the derivatives $\partial G^{a} / \partial x$. Substituting all of these into Eqs. (30b) gives the following expression for $G^{<}$in the device:

$$
\begin{aligned}
G^{<}(12) & =g_{D}^{<}(12)+g_{D}^{r}(1 L) \Sigma_{L}^{r} G^{<}(L 2)+g_{D}^{r}(1 L) \Sigma_{L}^{<} G^{a}(L 2) \\
& +g_{D}^{<}(1 L) \Sigma_{L}^{a} G^{a}(L 2)+(L \rightarrow R) \quad\left(L<x_{1}, x_{2}<R\right),
\end{aligned}
$$

where $\Sigma_{L, R}^{r}, \Sigma_{L, R}^{a}$ are given by Eqs. (16) and their complex conjugate, and

$$
\Sigma_{\alpha}^{<}(\omega)=\frac{g_{\alpha}^{<}(\alpha \alpha)}{g_{\alpha}^{r}(\alpha \alpha) g_{\alpha}^{a}(\alpha \alpha)} \quad(\alpha=L, R)
$$

In the usual case the potential in the leads $V_{L, R}$ is constant. Then, using Eqs. (7],27), this becomes equal to Eq. (26). Thus the physical argument used at the beginning of this section is correct, at least as long as the $\gamma^{\prime}$ s are real.

Eq. (31) is of the standard form for the analytic continuation of a Dyson's equation for $G^{r}$ to an equation for $G^{<}$. It is easy to show that Eq. (31) can be written equivalently as the Keldysh quantum kinetic equation

$$
G^{<}=\left(1+G^{r} \Sigma^{r}\right) g_{D}^{<}\left(1+\Sigma^{a} G^{a}\right)+G^{r} \Sigma^{<} G^{a}
$$

For a finite length device, the first term in Eq. (33) vanishes. 9.12 There are various ways to see this. It is clear on physical grounds that the exact Green's function including coupling to semi-infinite leads cannot depend on the initial occupancy of the finite device. We could choose $\mu_{D} \rightarrow-\infty$, in which case the device is initially unoccupied and the unperturbed $g_{D}^{<}$vanishes. Proof that the term vanishes is based on the fact that the unperturbed spectral function $A_{D}$ for a finite device is a sum of Dirac $\delta$-functions. The first term written symbolically in Eq. (33) is, in detail,

$$
\begin{aligned}
g_{D}^{<}(12) & +G^{r}(1 L) \Sigma_{L}^{r} g_{D}^{<}(L 2)+G^{r}(1 R) \Sigma_{R}^{r} g_{D}^{<}(R 2) \\
& +g_{D}^{<}(1 L) \Sigma_{L}^{a} G^{a}(L 2)+g_{D}^{<}(1 R) \Sigma_{R}^{a} G^{a}(R 2) \\
& +G^{r}(1 L) \Sigma_{L}^{r} g_{D}^{<}(L L) \Sigma_{L}^{a} G^{a}(L 2) \\
& +G^{r}(1 L) \Sigma_{L}^{r} g_{D}^{<}(L R) \Sigma_{R}^{a} G^{a}(R 2) \\
& +G^{r}(1 R) \Sigma_{R}^{r} g_{D}^{<}(R R) \Sigma_{R}^{a} G^{a}(R 2) \\
& +G^{r}(1 R) \Sigma_{R}^{r} g_{D}^{<}(R L) \Sigma_{L}^{a} G^{a}(L 2) .
\end{aligned}
$$


For the finite device we expand the unperturbed $g_{D}$ 's in discrete energy eigenstates $\psi_{i}(x)$ of $H_{D}$ with boundary conditions like Eq. (5]b,c):

$$
\begin{aligned}
& g_{D}^{r}(12)=\sum_{i} \frac{\psi_{i}\left(x_{1}\right) \psi_{i}\left(x_{2}\right)}{\hbar \omega-\varepsilon_{i}+i \eta} \\
& g_{D}^{<}(12)=-2 \pi f_{D}(\omega) \sum_{i} \psi_{i}\left(x_{1}\right) \psi_{i}\left(x_{2}\right) \delta\left(\hbar \omega-\varepsilon_{i}\right) .
\end{aligned}
$$

Since each term in Eq. (34) contains a factor of $g_{D}^{<}$, the term can be nonzero only if $\hbar \omega$ equals some eigenenergy $\varepsilon_{i}$. But in this case [and solving for $G^{r, a}(L 2), G^{r, a}(R 2)$ from Eq. (15)], some algebra shows that the terms in Eq. (34) cancel exactly.

Consequently the total NEGF is given by the second term indicated symbolically in Eq. (33). In detail for us this is

$$
G^{<}(12)=G^{r}(1 L) \Sigma_{L}^{<} G^{a}(L 2)+G^{r}(1 R) \Sigma_{R}^{<} G^{a}(R 2)
$$

It seems from this last equation that the scattering functions $\Sigma_{L, R}^{<}$must be independent of the original choice of internal boundary conditions. After all, $G^{r}, G^{a}$, and $G^{<}$are independent of this choice, and these three and $\Sigma^{<}$are related by Eq. (37). However, this conclusion is not quite correct. We have shown that the $\Sigma_{L, R}^{<}$are independent of the internal boundary conditions, and given by

$$
\Sigma_{\alpha}^{<}(\omega)=i f_{\alpha}(\omega) \frac{\hbar^{2} k_{\alpha}}{m} \theta\left(\hbar \omega-V_{\alpha}\right), \quad \alpha=L, R,
$$

as long as the $\gamma$ 's are all real. However, when any of the $\gamma$ are complex, a similar analysis shows that $\Sigma_{L, R}^{<}$pick up extra terms; but the extra terms give a cancelling contribution to Eq. (37). The accurate statement is rather that one can always choose the scattering functions to be of the form given by Eq. (38).

\section{SUMMARY}

In this paper we have analyzed the replacement of leads by coupling self-energies in calculations of nonequilibrium Green's functions. This was based on a modification of Feuchtwang's approach for continuum systems. In general the form of a self-energy depends on the way in which the unperturbed Green's functions are defined. The final Green's functions

of course do not. Here we investigated a class of homogeneous boundary conditions at the 
junctions between device and leads. Because these internal boundary conditions are completely arbitrary, the retarded self-energy $\Sigma^{r}$ can take any value. The "less-than" self-energy or scattering function $\Sigma^{<}$, on the other hand, can be taken to be entirely independent of the choice of boundary conditions.

In one dimension no particular choice of boundary conditions is easier than another. In more complicated calculations, for example of two- and three-dimensional devices with nonuniform cross-sections, the ideas to take away from this work are: (1) The leads can be replaced by self-energies; (2) All internal boundary conditions give the same final result; so (3) Choose the boundary conditions which make the calculation easy. Following this route in our opinion simplifies transport calculations in general electronic devices of arbitrary cross-section. We will explore this in a subsequent paper.10

\section{Acknowledgments}

We acknowledge support from the NSF through grant DMR99-72683.

* Electronic address: mjohnson@ucf.edu

1 L.P. Kadanoff and G. Baym, Quantum Statistical Mechanics (Benjamin, New York), 1962.

2 L.P. Keldysh, Sov. Phys. JETP 20, 1018.

3 D.C. Langreth, in Linear and Nonlinear Electron Transport in Solids, ed. by J.T. Devreese and E. Van Doren (Plenum, New York), 1976.

4 J. Rammer and H. Smith, Rev. Mod. Phys. 58, 323 (1986).

5 H. Haug and A.-P. Jauho, Quantum Kinetics in Transport and Optics of Semiconductors, Springer Series in Solid-State Sciences 123 (Springer-Verlag, Berlin), 1996.

6 Supriyo Datta, Electronic Transport in Mesoscopic Systems (Cambridge University Press, Cambridge), 1995.

7 C. Caroli, R. Combescot, P. Nozi'eres, and D. Saint-James, J. Phys. C 4, 916 (1971); C. Caroli, R. Combescot, P. Nozières, D. Lederer, and D. Saint-James, J. Phys. C 4, 2598 (1971); R. Combescot, J. Phys. C 4, 2611 (1971); C. Caroli, R. Combescot, P. Nozières, and D. SaintJames, J. Phys. C 5, 1972. 
8 T.E. Feuchtwang, Phys. Rev. B 10, 4121 and 4135 (1974); Phys. Rev. B 13, 517 (1976).

9 N.F. Schwabe, R.J. Elliott, and Ned S. Wingreen, Phys. Rev. B 54, 12953 (1996); C. Heide, R.J. Elliott, and Ned S. Wingreen, Phys. Rev. B 59, 4287 (1999).

10 Fredrick Michael and M.D. Johnson, unpublished.

11 Xindong Wang, J. Appl. Phys. 83, 6518 (1998).

12 R. Combescot, J. Phys. C 4, 2611 (1971). 